\title{
A Cross-Cultural Study on Own-Ethnicity Effects in the Relationship Between Body Dissatisfaction and Attentional Bias Towards Thin Bodies
}

House, T. ${ }^{\mathrm{a}, \mathrm{b}^{*}}$, Wong, H. K. ${ }^{c}$, Samuel, N. W. ${ }^{c}$, Stephen, I. D. ${ }^{d}$, Brooks, K. R. ${ }^{a}$, Bould, H. ${ }^{\mathrm{e}, \mathrm{f}, \mathrm{g}}$, Attwood, A. S. ${ }^{b, g}$, and Penton-Voak, I. S. ${ }^{b, h}$

School of Psychological Sciences, Faculty of Medicine, Health and Human Sciences, Macquarie University, Australia ${ }^{a}$

School of Psychological Science, University of Bristol, United Kingdom ${ }^{b}$ School of Psychology, University of Nottingham Malaysia, Malaysia ${ }^{c}$ Division of Psychology, Nottingham Trent University, United Kingdom ${ }^{d}$ Centre for Academic Mental Health, Population Health Sciences, Bristol Medical School, University of Bristol, United Kingdom ${ }^{\mathrm{e}}$

Gloucestershire Health and Care NHS Foundation Trust, Centre for Academic Mental Health, University of Bristol, United Kingdom ${ }^{f}$

MRC Integrative Epidemiology Unit, Bristol Medical School, University of Bristol, United Kingdom ${ }^{g}$ National Institute for Health Research Bristol Biomedical Research Centre, University Hospitals Bristol NHS Foundation Trust and University of Bristol, United Kingdom ${ }^{\mathrm{h}}$

*Corresponding author. Email address: thea.house@bristol.ac.uk 


\begin{abstract}
Studies suggest that an attentional bias to thin bodies is common amongst those with high levels of body dissatisfaction, which is a risk factor for, and symptom of, eating disorders. However, these studies have predominantly been conducted in Western countries with body stimuli involving images of White people. It is not known whether this relationship generalises to women from nonWestern countries, or whether it is influenced by ethnicity-congruency between body stimuli and participants. In a preregistered cross-cultural study, we recruited 150 Malaysian Chinese women and 150 White Australian women. To measure attentional bias to thin bodies, participants completed a dot probe task which presented images of women who self-identified their ethnicity as East Asian or as White Australian. Contrary to previous findings, we found no evidence for an association between body dissatisfaction and attentional bias to thin bodies. This lack of association was not affected by participant ethnicity (Malaysian Chinese versus White Australian) or ethnicity congruency between participants and body stimuli (own-ethnicity versus other-ethnicity). These results suggest that either the relationship between body dissatisfaction and attentional bias to thin bodies is not robust, or the dot probe task may not be a reliable measure of attentional bias to body size.
\end{abstract}

Keywords: attention, body dissatisfaction, body size, dot probe, cross-cultural comparison. 


\section{Introduction}

Body dissatisfaction is defined as the negative subjective evaluation of one's body and is often considered the attitudinal manifestation of body image disturbance (Cash and Deagle, 1997). Body dissatisfaction is a risk factor (Stice and Shaw, 2002) and symptom (American Psychiatric Association, 2013) of certain eating disorders, such as anorexia nervosa, making the construct a potential target for therapeutic intervention. High levels of body dissatisfaction are associated with multiple appearance-related cognitive biases (Rodgers and DuBois, 2016). For example, women reporting high levels of body dissatisfaction, in comparison to women with low levels of body dissatisfaction, tend to direct more attention towards thin bodies, as demonstrated by studies using eye-tracking (Stephen et al., 2018; Tobin et al., 2019) and dot probe tasks (Dondzilo et al., 2017; Joseph et al., 2016; Moussally et al., 2016) as measures of attentional bias. The dot probe task presents participants simultaneously with a thin body stimulus alongside a control stimulus (e.g., a non-thin body or a non-body object). Participants make speeded responses to a probe replacing one of the stimuli, and faster reaction times to probes replacing thin bodies compared to control stimuli are interpreted as reflecting an attentional bias towards thin bodies. Social comparison theory explains this relationship by suggesting women with high levels of body dissatisfaction make upward comparisons by attending to society's promoted ideal body size, which in Western societies is traditionally a thin body, and this comparison increases an individual's negative beliefs about their own body size (Myers and Crowther, 2009).

Although body image disturbance was once considered culturally bound to Western societies, research suggests that the globalisation of Western media has contributed to body dissatisfaction and adoption of the thin-ideal in many non-Western countries (Boothroyd et al., 2020; Swami et al., 2010). For example, cross-cultural research indicates that people in urban areas of Malaysia report similar body size preferences to people in Britain (Swami and Tovée, 2005). Further, Malaysian Chinese women have been shown to report greater body dissatisfaction than Australian women (Mellor et al., 2013). Previous dot probe studies investigating the relationship between body dissatisfaction and attentional bias towards thin bodies have been conducted in Western countries (Dondzilo et al., 2017; Joseph et al., 2016; Moussally et al., 2016), and therefore it is unknown whether the relationship generalises to non-Western populations. Ethnicity has previously been shown to moderate the effects of viewing idealised thin bodies on body dissatisfaction (DeBraganza and Hausenblas, 2010). Given the high levels of body dissatisfaction reported in Malaysian Chinese women (Mellor et al., 2013), we may expect a stronger relationship between body dissatisfaction and attentional bias to thin bodies in this population.

Most dot probe studies to date use White body stimuli (Dondzilo et al., 2017; Joseph et al., 2016; Moussally et al., 2016). Therefore, it is unknown whether the relationship between body dissatisfaction and attentional bias to thin bodies generalises beyond these stimuli. Any such relationship may be influenced by own-ethnicity bias, which was originally demonstrated as the tendency for people to be more accurate at identifying the faces of people of their own ethnicity, than those with other ethnicities (Meissner and Brigham, 2001; Wong et al., 2020). More specifically, own-ethnicity faces are more likely to capture a participant's attention than faces of another ethnicity (Hodsoll et al., 2010; Zhou et al., 2015). If a similar bias is present for own versus otherethnicity body stimuli, then we may expect the relationship between body dissatisfaction and attentional bias to be stronger when participants are responding to own-ethnicity compared to other-ethnicity body stimuli. This would be consistent with the suggestion in social comparison theory that people make comparisons with similar others (Myers and Crowther, 2009). 
We used a dot probe task to examine whether cross-cultural differences and own-ethnicity effects influenced the relationship between body dissatisfaction and attentional bias towards thin bodies in Malaysian Chinese and White Australian women. We hypothesised that (1) body dissatisfaction would be positively associated with attentional bias towards thin bodies, so participants with higher body dissatisfaction would have a greater attentional bias towards thin bodies; (2) the association between body dissatisfaction and attentional bias towards thin bodies would be stronger for Malaysian Chinese than White Australian participants; (3) the association would be stronger when participants responded to own-ethnicity compared to other-ethnicity body stimuli. The study protocol was preregistered on the Open Science Framework (https://osf.io/yt5fh/).

\section{Material and methods}

\section{Participants and recruitment}

We recruited 150 Malaysian Chinese and 150 White Australian participants, giving over $90 \%$ power to detect an effect size of $r=.26$ in each group (we reduced the effect size reported in Dondzilo et al. (2017) by 33\% to account for the overinflation of published effect sizes (Schäfer and Schwarz, 2019)). Participants were required to be 18-35 years old, female, and either White Australian (Australian sample) or Malaysian Chinese (Malaysian sample). White Australian participants were recruited via Macquarie University's study signup system and reimbursed with course credit. For the Malaysian Chinese sample, 83 participants were recruited via University of Nottingham Malaysia's study signup system (reimbursed with course credit) and 67 participants were recruited via social media adverts and snowball sampling (reimbursed with RM5 (approximately US\$1.20)).

\section{Measures}

\section{Body Dissatisfaction}

Body dissatisfaction was measured using a modified version of the body shape satisfaction scale (Pingitore et al., 1997). Participants rated their satisfaction with each of 18 parts or features of their body using a Likert scale ranging from 1-7 (1 representing "Very dissatisfied" and 7 representing "Very satisfied"). Responses for each item were reverse scored and a single body dissatisfaction score calculated for each participant by summing responses for all items. Potential scores ranged from 18 to 126, with higher scores representing greater body dissatisfaction. Cronbach's alpha for the scale with our total sample demonstrated excellent internal consistency $(\alpha=0.93)$.

\section{Stimuli}

Body stimuli were obtained from previous research conducted on women recruited in Australia who identified as East Asian or White Australian and provided written consent for their photographs to be used in future research (Gould-Fensom et al., 2019). Body stimuli selected for the present study constituted ten East Asian identities and ten White Australian identities, matched for body mass index (BMI). For each identity, the Spherize tool in Photoshop was used to create a version expanded horizontally by $50 \%$ and a version contracted horizontally by $50 \%$ to simulate higher and lower BMIs respectively (for more details, see Gould-Fensom et al., 2019). All body stimuli had faces covered with a black square to prevent any influence of facial characteristics (Figure 1). 


\section{Figure 1}

Example body stimuli

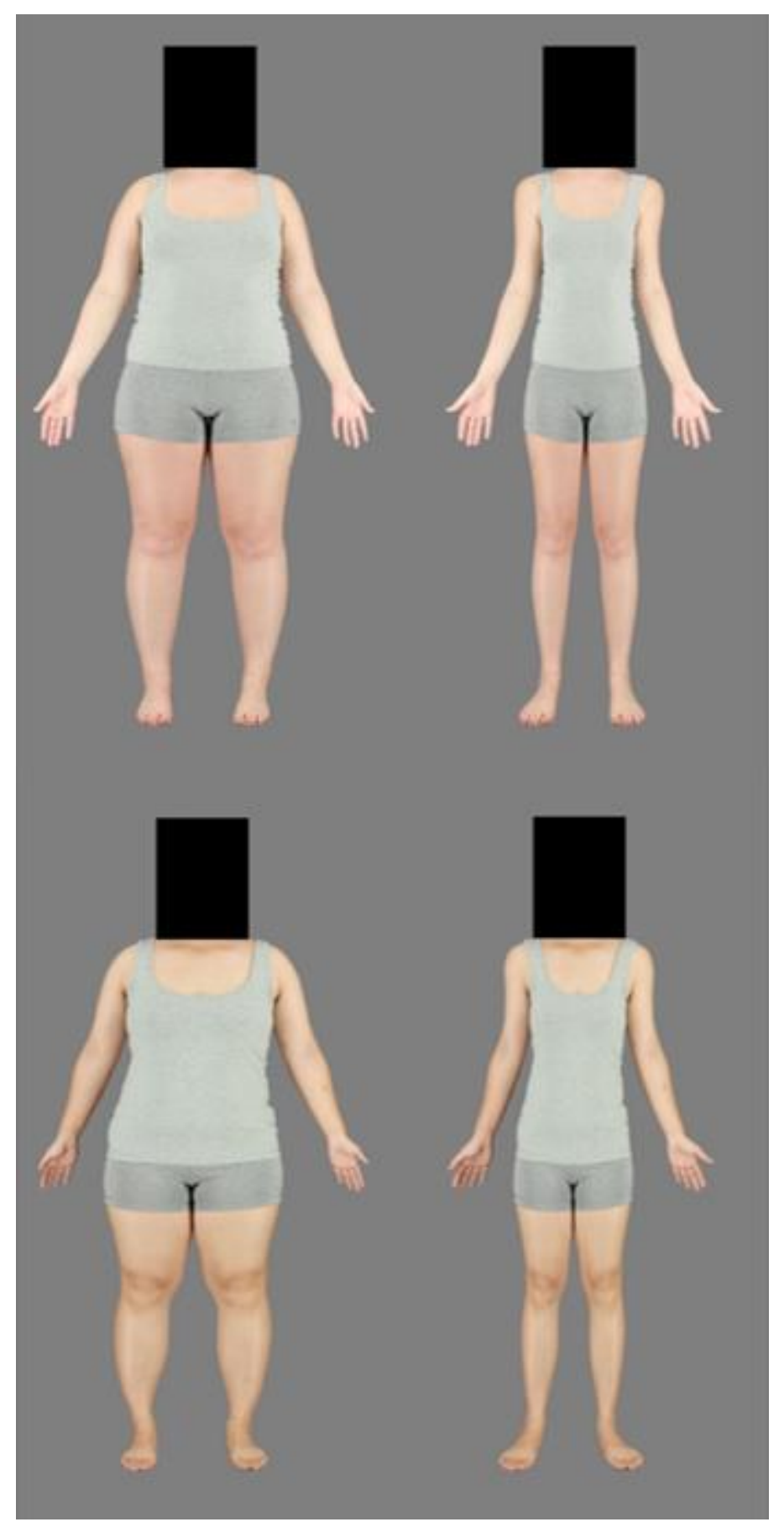

Note. These example body stimuli depict expanded (left) and contracted (right) versions of the same identities. Body stimuli on the top row are of a woman identifying as White Australian, while those on the bottom row are of a woman identifying as East Asian.

\section{Dot probe task}

Attentional bias was measured using a dot probe task based on the version used by Dondzilo et al. (2017). Each trial started with 1000 ms presentation of a fixation cross, followed by two body stimuli (one expanded and one contracted version of the same identity) presented simultaneously for $100 \mathrm{~ms}$ on either side of the fixation cross (sides randomised; Figure 2). The body stimuli then disappeared, and a probe appeared (either the letter " $p$ " or " $q$ ") in the location previously occupied 
by one of the body stimuli. The probe location was randomised, having an equal probability of replacing each body stimulus. Participants were required to identify the letter as quickly and accurately as possible by pressing the corresponding keyboard button (either " $p$ " or " $q$ ").

The dot probe task consisted of 320 trials divided into four blocks of 80, with a 30-second break between each block. Two blocks presented participants with East Asian body stimuli while the other two presented participants with White Australian body stimuli. The block order, and order of stimulus presentation within each block, was randomised for each participant. Following Dondzilo et al. (2017), we excluded trials when the participant responded incorrectly or when their reaction time was less than $200 \mathrm{~ms}$ or more than 2.5 standard deviations above their mean reaction time. Mean response times for the remaining trials were used to generate attentional bias scores using the following formula (MacLeod and Mathews, 1988):

Attentional bias score $=([\mathrm{LPRT}-\mathrm{LPLT}]+[\mathrm{RPLT}-\mathrm{RPRT}]) / 2$

Here, 'LPRT' refers to the mean response time when the probe $(P)$ was located in the left $(L)$ of the fixation cross but the contracted target body stimulus (T) was located in the right (R), and so on. $A$ positive attentional bias score represents a bias to contracted body stimuli while a negative attentional bias score represents a bias to expanded body stimuli.

Figure 2

Example dot probe trial

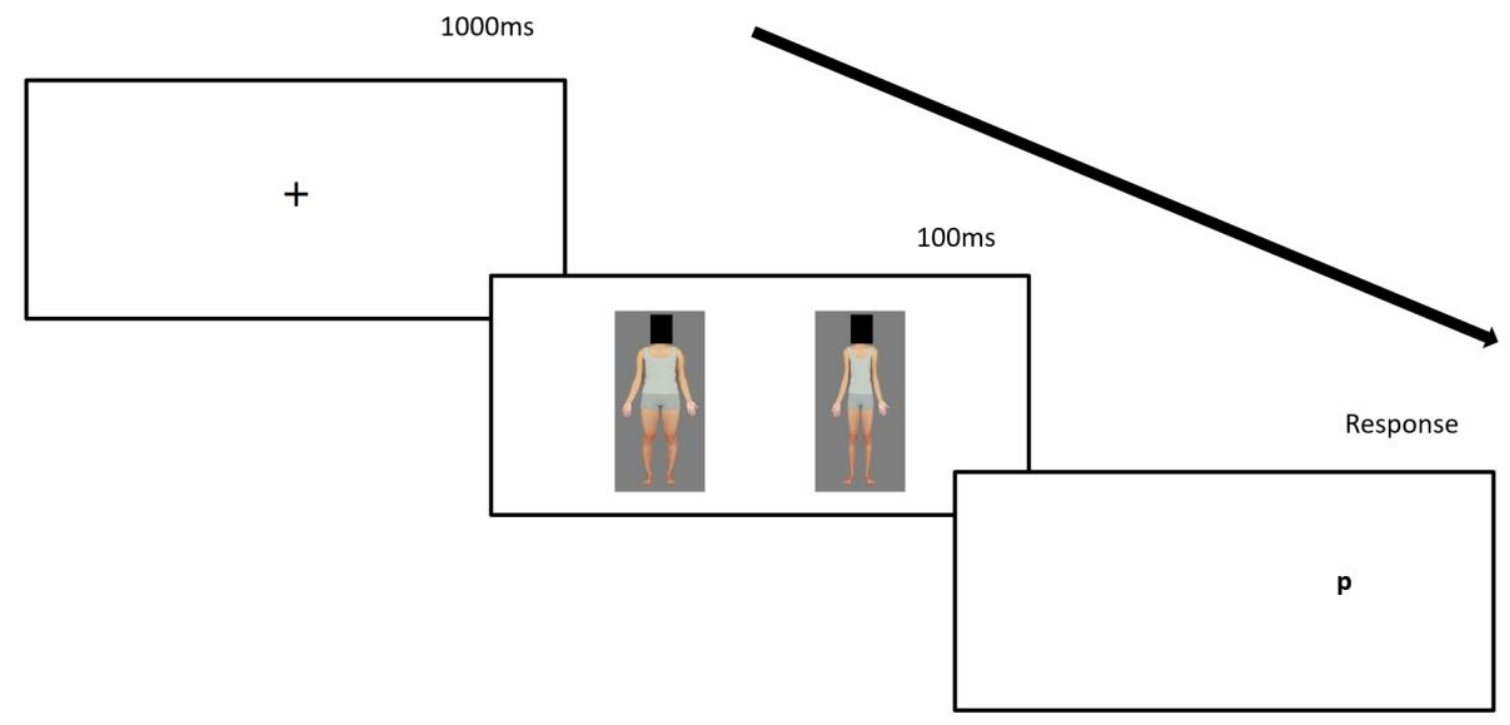

Note. In this example trial the body stimuli involved an expanded and a contracted version of the same East Asian woman, and the probe (letter "p") replaced the contracted target body.

\section{Procedure}

Participants provided informed consent and completed the study online via Gorilla

(https://gorilla.sc/; Anwyl-Irvine et al., 2020). A demographic questionnaire to determine eligibility (see Supplementary Materials) was followed by the body shape satisfaction scale, followed by 10 practice dot probe trials that were identical to the main dot probe trials, except that participants were presented with a green tick for responding correctly and a red cross for responding incorrectly. Body stimuli for the practice trials were selected at random from the pool of 20 identities. Participants then completed the main dot probe task, followed by a debrief and final consent form. 


\section{Data analysis}

We conducted three preregistered linear mixed effects models using the Ime4 R package. For model 1 , we ran a random intercepts model using the restricted maximum likelihood approach to predict attentional bias from the fixed effect of body dissatisfaction, including age and BMI as confounding fixed effects and participant ID as a random effect. We group mean centred the variables body dissatisfaction, age, and BMI and we estimated $p$ values using the Satterthwaite's degrees of freedom method with the ImerTest R package. We inferred support for Hypothesis 1 if body dissatisfaction had a positive coefficient $(p<.05)$.

For model 2, participant ethnicity was dummy coded (Malaysian Chinese $=0$ and White Australian $=$ 1) and added to model 1 as a fixed effect to test for an interaction with body dissatisfaction. We inferred support for Hypothesis 2 if there was an interaction between body dissatisfaction and participant ethnicity $(p<.05)$ and follow-up simple slope analyses demonstrated larger coefficients for Malaysian Chinese participants than White Australian participants. For model 3, ethnicity congruency was dummy coded (other-ethnicity $=0$ and own-ethnicity $=1$ ) and added to the model 2 as a fixed effect to test for an interaction with body dissatisfaction. We inferred support for Hypothesis 3 if there was an interaction between body dissatisfaction and ethnicity congruency $(p<$ .05 ) and if follow-up simple slope analyses demonstrated larger coefficients for own-ethnicity stimuli than other-ethnicity stimuli.

We conducted further exploratory analyses that were not pre-registered. First, we conducted three likelihood ratio tests to compare the likelihood of the models using analysis of variance and a criterion of $p<.05$. We compared model 1 to the null model (i.e. model 1 without body dissatisfaction included as a fixed effect), model 2 to model 1 , and model 3 to model 2 . Second, we used bivariate correlations to test the relationship between body dissatisfaction and attentional bias to contracted bodies separately for each participant group and ethnicity congruency condition. We also conducted Bayesian correlation tests using the R package BayesFactor to determine the likelihood of the alternative hypotheses $(r \neq 0)$ in relation to the corresponding null hypotheses $(r=$ $0)$.

\section{Results}

Two Malaysian Chinese participants and one White Australian participant responded correctly on fewer than $60 \%$ of the dot probe trials. They were excluded and replaced. Participant characteristics are presented in Table 1.

The results of the linear mixed effects models are presented in Table 2. Model 1 found no evidence for an association between body dissatisfaction and attentional bias to contracted bodies. A likelihood ratio test found no evidence for model 1 being a better fit than the null model $\left(\chi^{2}(1)=\right.$ $1.68, p=.196)$. Model 2 found no evidence for an interaction between body dissatisfaction and participant ethnicity on attentional bias to contracted bodies. A likelihood ratio test found no evidence for model 2 being a better fit than model $1\left(\chi^{2}(2)=1.74, p=.418\right)$. Therefore, we did not conduct simple slope analyses or test for a three-way interaction between body dissatisfaction, participant ethnicity, and ethnicity congruency in model 3 . Model 3 found no evidence for an interaction between body dissatisfaction and ethnicity congruency on attentional bias to contracted bodies. A likelihood ratio test found no evidence for model 3 being a better fit than model $2\left(\chi^{2}(2)=\right.$ $0.08, p=.959)$. Therefore, we did not conduct follow-up simple slope analyses. The Pearson's correlation coefficients and Bayes factors for the relationship between body dissatisfaction and attentional bias to contracted bodies are reported in Table 3. The correlation coefficients did not provide evidence of an association between body dissatisfaction and attentional bias to contracted 
bodies for any of the participant ethnicity groups or ethnicity congruency conditions. The Bayes factors provided moderate support for the null hypothesis $(r=0)$ for each participant ethnicity group and ethnicity congruency condition (Lee and Wagenmakers, 2014).

\section{Table 1}

The descriptive statistics for the participant characteristics

\begin{tabular}{lllll}
\hline & \multicolumn{2}{c}{ Malaysian Chinese $(N=150)$} & \multicolumn{2}{c}{ White Australian $(N=150)$} \\
\cline { 2 - 5 } & $M d n$ & $I Q R$ & $M d n$ & $I Q R$ \\
\hline Age (years) & 22.00 & 5.00 & 18.00 & 4.00 \\
Body mass index (BMI) & 19.72 & 4.16 & 22.50 & 6.55 \\
Body dissatisfaction & 73.00 & 23.00 & 73.50 & 28.50 \\
Attentional bias score to own-ethnicity body stimuli & 1.46 & 28.14 & 2.17 & 27.80 \\
Attentional bias score to other-ethnicity body stimuli & 0.01 & 22.74 & -0.67 & 27.52 \\
Dot probe accuracy (percentage of correct trials) & 96.88 & 4.61 & 95.31 & 5.00 \\
\hline
\end{tabular}

Note. We have reported the median $(M d n)$ and interquartile range $(I Q R)$ due to the non-normal distribution of some variables.

Table 2

The results of the three linear mixed effects models with the outcome variable as attentional bias score $(N=300)$

\begin{tabular}{lllllllllll}
\hline & \multicolumn{3}{c}{ Model 1 } & \multicolumn{3}{c}{ Model 2 } & \multicolumn{3}{c}{ Model 3 } \\
\cline { 2 - 11 } Effect & B & SE & $p$ & $B$ & SE & $p$ & $B$ & SE & $p$ \\
\hline Body dissatisfaction & 0.10 & 0.08 & .197 & 0.15 & 0.11 & .152 & 0.17 & 0.13 & .192 \\
Age & -0.17 & 0.34 & .621 & -0.16 & 0.34 & .624 & -0.16 & 0.34 & .625 \\
Body mass index (BMI) & -0.04 & 0.33 & .915 & -0.02 & 0.33 & .950 & -0.02 & 0.33 & .950 \\
Participant ethnicity & - & - & - & -3.18 & 2.88 & .270 & -3.18 & 2.89 & .271 \\
Body dissatisfaction * participant ethnicity & - & - & - & -0.11 & 0.15 & .475 & -0.11 & 0.15 & .476 \\
Ethnicity congruency & - & - & - & - & - & - & -0.53 & 2.89 & .855 \\
Body dissatisfaction * ethnicity congruency & - & - & - & - & - & - & -0.03 & 0.15 & .824 \\
\hline
\end{tabular}

Table 3

The correlation coefficients and Bayes factors for the relationship between body dissatisfaction and attentional bias score $(N=300)$

\begin{tabular}{llllll}
\hline Participant ethnicity & Ethnicity congruency & $\mathrm{df}$ & $r$ & $p$ & $\mathrm{BF}_{10}$ \\
\hline Malaysian Chinese & Own-ethnicity & 148 & 0.07 & .391 & 0.27 \\
Malaysian Chinese & Other-ethnicity & 148 & 0.07 & .412 & 0.26 \\
White Australian & Own-ethnicity & 148 & 0.03 & .695 & 0.21 \\
White Australian & Other-ethnicity & 148 & 0.04 & .603 & 0.22 \\
\hline
\end{tabular}

\section{Discussion}

The results of this study did not support any of our pre-registered hypotheses. We found no evidence for an association between body dissatisfaction and attentional bias to thin bodies, as measured on a dot probe task. This absence of association was not altered by participant ethnicity (Malaysian Chinese versus White Australian) or the ethnicity congruency of the body stimuli in the 
dot probe task (own-ethnicity versus other-ethnicity). The absence of association between body dissatisfaction and attentional bias contrasts with previous dot probe studies that found evidence for an association in women in Australia (Dondzilo et al., 2017), the United States (Joseph et al., 2016), and Switzerland (Moussally et al., 2016). The absence of an association also contrasts with studies using eye-tracking as a measure of attentional bias (e.g. Stephen et al., 2018; Tobin et al., 2019).

One possible explanation for this inconsistency is that we conducted our dot probe task online, as opposed to face to face in a laboratory setting like the aforementioned studies. Therefore, participants in our study may have experienced reduced motivation and more distractions. However, a recent dot probe study conducted in a laboratory setting in Australia also failed to replicate this relationship (House et al., 2021), indicating that the experimental setting is unlikely to explain this discrepancy. Another possible explanation is that the association is dependent on the type of control stimuli used during the dot probe task. Dondzilo et al. (2017) used an abstract art image as their control stimulus, so participants may have demonstrated an attentional bias to bodies generally, rather than thin bodies in particular. In our study we used an expanded body as the control stimulus; therefore, the thin body may not have been visually contrasting enough to capture the participants' attention. However, Joseph et al. (2016) and Moussally et al. (2016) found evidence for this association using larger bodies as their control stimuli; therefore, it seems unlikely that our choice of control stimuli explains the inconsistency.

Another potential explanation may be that the relationship depends on the stimulus-onset asynchrony (SOA) of the dot probe task, which refers to the interval between the onset of the body stimuli and the onset of the probe. Studies reporting a relationship between body dissatisfaction and attentional bias used an SOA of 500ms (Dondzilo et al., 2017; Joseph et al., 2016; Moussally et al., 2016), whereas we used an SOA of $100 \mathrm{~ms}$. Moussally et al. (2016) suggested that, in dot probe tasks, short SOAs (e.g. 100ms) measure facilitated attention, whereas medium length SOAs (e.g. 500ms) measure delayed disengagement. They found people with high body dissatisfaction demonstrated an attentional bias to thin bodies using an SOA of $500 \mathrm{~ms}$ but not $100 \mathrm{~ms}$; therefore, our results are consistent with body dissatisfaction being related to delayed disengagement from thin bodies but not facilitated attention. However, House et al. (2021) used a dot probe task with an SOA of 500ms and found no evidence for a relationship between body dissatisfaction and attentional bias to thin bodies. Further, other reaction-time based tasks thought to measure delayed disengagement do not provide evidence for a relationship between body dissatisfaction and delayed disengagement bias from thin bodies (Dondzilo et al., 2021). Chapman et al. (2019) suggested that longer SOAs reduce the reliability of the dot probe task, because participants have more time to redistribute their attention before responding to the probe. Therefore, the variability in findings may be a consequence of the poor reliability of the dot probe task as a measure of attentional bias. This interpretation is consistent with previous research highlighting the task's poor internal consistency and test-retest reliability (Schmukle, 2005).

To the best of our knowledge, our study is the first to use a dot probe task to investigate crosscultural differences and own-ethnicity effects on the relationship between body dissatisfaction and attentional bias to thin bodies. We did not find evidence to suggest the association was affected by participant ethnicity or the ethnicity congruency of the body stimuli. Also, our results found no evidence of an association between body dissatisfaction and attention, suggesting that the dot probe task may not be a reliable measure of attentional bias to body size. Thus, great caution must be applied before ruling out own-ethnicity effects and group differences between White Australian and Malaysian Chinese women. Free viewing eye-tracking paradigms are thought to be a more 
reliable measure of attentional bias (e.g. Skinner et al., 2018) and have consistently produced evidence for a relationship between body dissatisfaction and attentional bias to thin bodies (e.g. Stephen et al., 2018; Tobin et al., 2019). Therefore, future research may employ eye-tracking techniques to investigate the moderating effects of ethnicity and ethnicity-congruency on the relationship between body dissatisfaction and attentional bias to body size.

\section{Ethical Statement}

Ethical approval to conduct this research was obtained from Macquarie University Human Research Ethics Committee (reference 52020573222184) and the University of Nottingham Malaysia Science \& Engineering Research Ethics Committee (SEREC; reference NWYS101120). Informed consent was obtained from all participants who took part in the experiments.

\section{Data availability}

The study materials, analysis code, and data for the participants recruited in Australia are publicly available from the University of Bristol Research Data Repository (DOI: TBC after peer review). For the participants recruited in Malaysia, we did not obtain explicit consent for data sharing. Therefore, we are unable to share the data for these participants due to ethical concerns.

\section{Funding source}

This work was supported by a Macquarie University Research Training Pathway scholarship and the Industrial and International Leverage Fund (IILF) as part of the Engineering and Physical Sciences Research Council (EPSRC) Doctoral Training Partnership (DTP). 


\section{References}

American Psychiatric Association, 2013. Diagnostic and Statistical Manual of Mental Disorders, 5th ed.

Anwyl-Irvine, A.L., Massonnié, J., Flitton, A., Kirkham, N., Evershed, J.K., 2020. Gorilla in our midst: An online behavioral experiment builder. Behav. Res. Methods 52, 388-407.

https://doi.org/10.3758/s13428-019-01237-x

Boothroyd, L.G., Jucker, J.L., Thornborrow, T., Barton, R.A., Burt, D.M., Evans, E.H., Jamieson, M.A., Tovée, M.J., 2020. Television consumption drives perceptions of female body attractiveness in a population undergoing technological transition. J. Pers. Soc. Psychol. 119, 839-860.

https://doi.org/10.1037/pspi0000224

Cash, T.F., Deagle, E.A., 1997. The Nature and Extent of Body-Image Disturbances in Anorexia Nervosa and Bulimia Nervosa: A Meta-Analysis. Int. J. Eat. Disord. 22, 107-126.

https://doi.org/10.1002/(SICI)1098-108X(199709)22:2<107::AID-EAT1>3.0.CO;2-J

Chapman, A., Devue, C., Grimshaw, G.M., 2019. Fleeting reliability in the dot-probe task. Psychol. Res. 83, 308-320. https://doi.org/10.1007/s00426-017-0947-6

DeBraganza, N., Hausenblas, H.A., 2010. Media Exposure of the Ideal Physique on Women's Body Dissatisfaction and Mood: The Moderating Effects of Ethnicity. J. Black Stud. 40, 700-716. https://doi.org/10.1177/0021934708317723

Dondzilo, L., Basanovic, J., Grafton, B., Bell, J., Turnbull, G., MacLeod, C., 2021. A serial mediation model of attentional engagement with thin bodies on body dissatisfaction: The role of appearance comparisons and rumination. Curr. Psychol. https://doi.org/10.1007/s12144-021-01574-1

Dondzilo, L., Rieger, E., Palermo, R., Byrne, S., Bell, J., 2017. The mediating role of rumination in the relation between attentional bias towards thin female bodies and eating disorder symptomatology. PloS One 12, e0177870. https://doi.org/10.1371/journal.pone.0177870

Gould-Fensom, L., Tan, C.B.Y., Brooks, K.R., Mond, J., Stevenson, R.J., Stephen, I.D., 2019. The Thin White Line: Adaptation Suggests a Common Neural Mechanism for Judgments of Asian and Caucasian Body Size. Front. Psychol. 10, 1-9. https://doi.org/10.3389/fpsyg.2019.02532

Hodsoll, J., Quinn, K.A., Hodsoll, S., 2010. Attentional prioritization of infant faces is limited to ownrace infants. PloS One 5, 1-5. https://doi.org/10.1371/journal.pone.0012509

House, T., Stephen, I.D., Penton-Voak, I.S., Brooks, K.R., 2021. The Effect of Attention on Body Size Adaptation and Body Dissatisfaction. PsyArxiv. https://doi.org/10.31234/osf.io/y9s7c

Joseph, C., LoBue, V., Rivera, L.M., Irving, J., Savoy, S., Shiffrar, M., 2016. An attentional bias for thin bodies and its relation to body dissatisfaction. Body Image 19, 216-223.

https://doi.org/10.1016/j.bodyim.2016.10.006

Lee, M.D., Wagenmakers, E.J., 2014. Bayesian cognitive modeling: A practical course. Cambridge university press.

MacLeod, C., Mathews, A., 1988. Anxiety and the Allocation of Attention to Threat. Q. J. Exp.

Psychol. Sect. A 40, 653-670. https://doi.org/10.1080/14640748808402292 
Meissner, C.A., Brigham, J.C., 2001. Thirty years of investigating the own-race bias in memory for faces: A meta-analytic review. Psychol. Public Policy Law 7, 3-35.

https://doi.org/10.1037/1076-8971.7.1.3

Mellor, D., Waterhouse, M., Mamat, N.H. bt, Xu, X., Cochrane, J., McCabe, M., Ricciardelli, L., 2013. Which body features are associated with female adolescents' body dissatisfaction? A cross-cultural study in Australia, China and Malaysia. Body Image 10, 54-61.

https://doi.org/10.1016/j.bodyim.2012.10.002

Moussally, J.M., Brosch, T., Van der Linden, M., 2016. Time course of attentional biases toward body shapes: The impact of body dissatisfaction. Body Image 19, 159-168.

https://doi.org/10.1016/j.bodyim.2016.09.006

Myers, T.A., Crowther, J.H., 2009. Social comparison as a predictor of body dissatisfaction: A metaanalytic review. J. Abnorm. Psychol. 118, 683-698. https://doi.org/10.1037/a0016763

Pingitore, R., Spring, B., Garfieldt, D., 1997. Gender Differences in Body Satisfaction. Obes. Res. 5, 402-409. https://doi.org/10.1002/j.1550-8528.1997.tb00662.x

Rodgers, R.F., DuBois, R.H., 2016. Cognitive biases to appearance-related stimuli in body dissatisfaction: A systematic review. Clin. Psychol. Rev. 46, 1-11.

https://doi.org/10.1016/j.cpr.2016.04.006

Schäfer, T., Schwarz, M.A., 2019. The Meaningfulness of Effect Sizes in Psychological Research: Differences Between Sub-Disciplines and the Impact of Potential Biases. Front. Psychol. 10, 813. https://doi.org/10.3389/fpsyg.2019.00813

Schmukle, S.C., 2005. Unreliability of the dot probe task. Eur. J. Personal. 19, 595-605. https://doi.org/10.1002/per.554

Skinner, I.W., Hübscher, M., Moseley, G.L., Lee, H., Wand, B.M., Traeger, A.C., Gustin, S.M., McAuley, J.H., 2018. The reliability of eyetracking to assess attentional bias to threatening words in healthy individuals. Behav. Res. Methods 50, 1778-1792. https://doi.org/10.3758/s13428-017-0946-y

Stephen, I.D., Sturman, D., Stevenson, R.J., Mond, J., Brooks, K.R., 2018. Visual attention mediates the relationship between body satisfaction and susceptibility to the body size adaptation effect. PloS One 13, e0189855. https://doi.org/10.1371/journal.pone.0189855

Stice, E., Shaw, H.E., 2002. Role of body dissatisfaction in the onset and maintenance of eating pathology: A synthesis of research findings. J. Psychosom. Res. 53, 985-993.

https://doi.org/10.1016/S0022-3999\%2802\%2900488-9

Swami, V., Frederick, D.A., Aavik, T., Alcalay, L., Allik, J., Anderson, D., Andrianto, S., Arora, A., Brännström, A., Cunningham, J., Danel, D., Doroszewicz, K., Forbes, G.B., Furnham, A., Greven, C.U., Halberstadt, J., Hao, S., Haubner, T., Hwang, C.S., Inman, M., Jaafar, J.L., Johansson, J., Jung, J., Keser, A., Kretzschmar, U., Lachenicht, L., Li, N.P., Locke, K., Lönnqvist, J.-E., Lopez, C., Loutzenhiser, L., Maisel, N.C., McCabe, M.P., McCreary, D.R., McKibbin, W.F., Mussap, A., Neto, F., Nowell, C., Alampay, L.P., Pillai, S.K., Pokrajac-Bulian, A., Proyer, R.T., Quintelier, K., Ricciardelli, L.A., RozmusWrzesinska, M., Ruch, W., Russo, T., Schütz, A., Shackelford, T.K., Shashidharan, S., Simonetti, F., Sinniah, D., Swami, M., Vandermassen, G., van Duynslaeger, M., Verkasalo, M., Voracek, M., Yee, C.K., Zhang, E.X., Zhang, X., Zivcic-Becirevic, I., 2010. The attractive female body weight and female body dissatisfaction in 26 countries across 10 world regions: results of the international body project I. Pers. Soc. Psychol. Bull. 36, 309-325. https://doi.org/10.1177/0146167209359702 
Swami, V., Tovée, M.J., 2005. Female physical attractiveness in Britain and Malaysia: A cross-cultural study. Body Image 2, 115-128. https://doi.org/10.1016/j.bodyim.2005.02.002

Tobin, L.N., Barron, A.H., Sears, C.R., von Ranson Kristin M, 2019. Greater body appreciation moderates the association between maladaptive attentional biases and body dissatisfaction in undergraduate women. J. Exp. Psychopathol. 10. https://doi.org/10.1177/2043808719838937

Wong, H.K., Stephen, I.D., Keeble, D.R.T., 2020. The Own-Race Bias for Face Recognition in a Multiracial Society. Front. Psychol. 11, 208. https://doi.org/10.3389/fpsyg.2020.00208

Zhou, G., Cheng, Z., Yue, Z., Tredoux, C., He, J., Wang, L., 2015. Own-race faces capture attention faster than other-race faces: evidence from response time and the N2pc. PloS One 10, 1-15. https://doi.org/10.1371/journal.pone.0127709 